\title{
Statin-centric versus low-density lipoprotein-centric approach for atherosclerotic cardiovascular disease prevention: a Singapore perspective
}

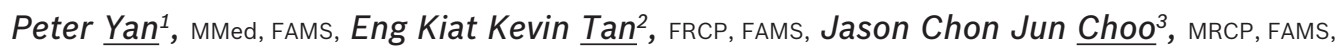
Choon Fong Stanley $\underline{\text { Liew }}^{4}$, MRCP, FAMS, Titus $\underline{\text { Lau }}^{5}$, FRCP, FAMS, David D $\underline{\text { Waters }}^{6}$, MD

\begin{abstract}
The link between cholesterol levels and atherosclerotic cardiovascular disease (ASCVD) is well-established. In Singapore, there is an increasing prevalence of risk factors for ASCVD. Like many Asian countries, Singapore's population is rapidly ageing and increasingly sedentary, which predisposes individuals to chronic health problems. Current international and local guidelines recommend statin therapy for the primary and secondary prevention of ASCVD. However, despite the effectiveness of statin therapy, some studies have highlighted that Asian patients with cardiovascular disease are not achieving target lipid goals. Furthermore, it is widely believed that the responses of Asians (both patients and physicians) to statin therapy are different from those of their Western counterparts. Experts convened in 2014 to determine the impact of current guidelines on clinical practice in Singapore. This review summarises the key findings and recommendations of these guidelines, and presents key principles to aid clinicians to manage the cardiovascular risk of their patients more effectively.
\end{abstract}

Keywords: atherosclerosis, cardiovascular disease, lipids, Singapore

\section{INTRODUCTION}

Southeast Asia (SEA) bears a significant proportion of the global burden of cardiovascular disease (CVD), a burden that appears to be increasing across the region. ${ }^{(1-3)}$ In 2011, a World Health Organization report estimated a cardiovascular-related mortality rate of 3.7 million within SEA, with ischaemic heart disease and stroke accounting for the majority of CVD deaths. ${ }^{(4)}$ The Asian region comprises a very heterogeneous population, which is evident in the differences in atherosclerotic cardiovascular disease (ASCVD)-related mortality reported across the region. ${ }^{(5)}$ Agestandardised mortality rates for stroke are elevated in East Asian countries and generally higher than those reported in Western countries. ${ }^{(6)}$ Although East Asian countries have lower coronary heart disease (CHD) mortality rates than Western countries, the age-standardised mortality rates for CHD in South Asian and some SEA countries are comparable to or higher than those of Western countries. ${ }^{(7)}$

The link between cholesterol levels and ASCVD is well-established. ${ }^{(2,3)}$ Major risk factors for ASCVD include hypercholesterolaemia, hypertension, diabetes mellitus, chronic kidney disease (CKD), obesity and metabolic syndrome. Lifestyle, including smoking, physical inactivity, and excessive dietary consumption of fats (especially trans fat) and carbohydrates, is also a major contributor to the development of ASCVD. ${ }^{(2)}$

Risk factors for CVD, such as diabetes mellitus and obesity, have been increasing in Asia. ${ }^{(8)}$ Singapore, often described as a microcosm of Asia as a whole, has a rapidly ageing and increasingly sedentary population, which predisposes individuals to chronic health problems faced across SEA. ${ }^{(5)}$ The prevalence of obesity in Singapore is predicted to increase fourfold, from $4.3 \%$ in 1990 to $15.9 \%$ in 2050 . This increase is expected to be paralleled by an increase in type 2 diabetes mellitus (diagnosed and undiagnosed) from $7.3 \%$ in 1990 to $15.0 \%$ in $2050 .{ }^{(6)}$ It has also been predicted that ethnic Indians and Malays will bear a disproportionate burden of disease compared with the Chinese. ${ }^{(5)}$

Experts convened in August 2014 to define the landscape of ASCVD prevention and management in Singapore, and to determine the impact of current guidelines on clinical practice in Singapore. This review summarises the key findings and recommendations of these guidelines, and presents key principles to help clinicians manage the cardiovascular risk of their patients more effectively.

\section{UNMET NEEDS IN THE PREVENTION OF ASCVD: A SINGAPORE PERSPECTIVE}

In SEA, ageing and population growth are projected to result in an increase in CVD of more than 50\% over the next two decades. Furthermore, projected unfavourable trends in blood pressure, total cholesterol, diabetes mellitus and body mass index may accelerate this epidemic. ${ }^{(9)}$ Overall, ageing will prolong exposure to CVD risk factors and increase the CVD burden as well as ASCVD-related mortality and morbidity.

The Asia Pacific Cohort Studies Collaboration, a meta-analysis of prospective observational studies in the Asia Pacific region

\footnotetext{
${ }^{1}$ Peter Yan Cardiology Clinic, Gleneagles Hospital, ${ }^{2}$ Kevin Tan Clinic for Diabetes, Thyroid and Hormones Pte Ltd, Mount Elizabeth Medical Centre, ${ }^{3}$ Department of Renal Medicine, Singapore General Hospital, ${ }^{4}$ Raffles Diabetes and Endocrine Centre, Raffles Hospital, ${ }^{5}$ Division of Nephrology, National University Health System, National University Hospital, Singapore, ${ }^{6}$ Department of Medicine, University of California, San Francisco, USA

Correspondence: Dr Peter Yan, Consultant Cardiologist, Peter Yan Cardiology Clinic, Gleneagles Medical Centre, 6 Napier Road, Suite \#04-10, Singapore 258499. peteryan@cardiology.com.sg
} 
(29 cohorts with almost two million person-years of follow-up of 352,033 individuals), found a continuous positive association between usual cholesterol levels and risk for CHD, which persisted after adjustment for age, gender, blood pressure and smoking. ${ }^{(10)}$ Each increase of $1 \mathrm{mmol} / \mathrm{L}$ in cholesterol level equated to approximately a $35 \%$ increase in risk of coronary death. The study found a weak positive association between cholesterol and combined risk of both fatal and nonfatal stroke ( $7 \%$ increase per $1 \mathrm{mmol} / \mathrm{L}$ ), but no association between total cholesterol level and stroke mortality. ${ }^{(10)}$

Numerous studies have shown that statin therapy effectively reduces low-density lipoprotein cholesterol (LDL-C) in Asian patients. ${ }^{(11-16)}$ However, despite the reduction of LDL-C following statin therapy, residual cardiovascular risk remains high, as illustrated by the Return on Expenditure Achieved for Lipid Therapy in Asia (REALITY-Asia) study. ${ }^{(17)}$ A retrospective cohort study conducted in China, Korea, Malaysia, Singapore, Taiwan and Thailand (comprising 437 physicians, 41\% of whom were cardiologists), REALITY-Asia recruited adults with hypercholesterolaemia who were newly initiated on statin monotherapy in order to examine treatment patterns, goal attainment and factors influencing treatment. The study found that undertreatment of patients occurred mostly in the highestrisk group; high-risk individuals with established $\mathrm{CHD}$ /diabetes mellitus were least likely to achieve LDL-C targets (38\% vs. $48 \%$ overall goal attainment). Despite considerable baseline cardiovascular risk, $89 \%$ of patients received statins at low or moderate equipotent doses; the majority were using lowintensity statins. ${ }^{(17)}$ The REALITY-Asia findings highlighted the presence of statin phobia at the regional, country, physician and patient levels. ${ }^{(17)}$ Factors associated with a significantly increased likelihood of goal attainment included advanced age and treatment with high-potency statins, whereas increased cardiovascular risk for $\mathrm{CHD}$ /diabetes mellitus at baseline (as compared with less than two risk factors) and baseline LDL-C levels were inversely associated with LDL-C goal achievement. ${ }^{(17)}$

In Singapore, approximately $47 \%$ of patients used lowpotency statins, the majority of which were prescribed by general practitioners; of these patients, $45 \%$ required up-titration. ${ }^{(18)}$ In particular, $40 \%-60 \%$ of diabetic patients required up-titration, but there was reluctance to up-titrate or switch to a higher dose or higher potency statin. A number of studies have highlighted that Asian patients with CVD are not achieving their target lipid goals despite statin therapy. ${ }^{(19-21)}$ Data from the Achievement in Singapore of Cholesterol Targets study confirmed that, in a cohort of over 5,000 CHD patients, most who were considered to be at high risk did not achieve the recommended LDL-C targets. ${ }^{(19)}$

\section{ASSESSMENT AND TREATMENT OF ASCVD RISK \\ Lipid profile}

While the 2013 American College of Cardiology/American Heart Association (ACC/AHA) Cholesterol Treatment Guidelines ${ }^{(22)}$ still use LDL-C levels to classify the degrees of risk for the primary prevention of ASCVD, the updated 2014 National Institute for
Health and Care Excellence (NICE) guidelines recommend the use of non-high-density lipoprotein cholesterol (non-HDL-C), as it is a better predictor of risk than LDL-C. ${ }^{(23)}$ Specifically, the NICE guidelines recommend the measurement of non-fasting lipid profile, namely total cholesterol, HDL-C, non-HDL-C and triglycerides, before the initiation of lipid modification therapy. Treatment recommendations are, however, based on statin intensity rather than LDL-C or non-HDL-C targets. ${ }^{(23)}$ When a target reduction of $>40 \%$ non-HDL-C is required, high-intensity statin therapy should be initiated. Although the definitions of low-, moderate- and high-intensity statin therapy are still based on estimations of LDL-C reduction, they differ slightly between the ACC/AHA and NICE guidelines (Table I). ${ }^{(22,23)}$ It should also be noted that the only recommended high-intensity statins are atorvastatin and rosuvastatin. For moderate- and low-intensity statin therapy, simvastatin, fluvastatin, lovastatin, pravastatin and pitavastatin together with lower doses of atorvastatin and rosuvastatin (only for moderate intensity) are recommended. Atorvastatin and rosuvastatin do not have a recommendation for low-intensity therapy.

\section{ASCVD risk scoring}

The 2013 ACC/AHA cholesterol guidelines adopt a broader focus on ASCVD risk (including CHD and stroke). The Pooled Cohort Equations risk calculator was used to assess the ten-year risk. ${ }^{(22)}$ These guidelines identify four groups of patients who will benefit from statin treatment: patients who have (a) clinical ASCVD (i.e. history of myocardial infarction [MI], stable angina, acute coronary syndrome [ACS], stroke, transient ischaemic attack, peripheral vascular disease) - secondary prevention; (b) familial hypercholesterolaemia with LDL-C $\geq 190 \mathrm{mg} / \mathrm{dL}$ - primary prevention; (c) diabetes mellitus (age 40-75 years) with LDL-C of 70-189 mg/dL - primary prevention; and (d) LDL-C of 70-189 mg/dL with a ten-year ASCVD risk $\geq 7.5 \%$ - primary prevention.

The NICE guidelines also advocate initiating statin therapy based on CVD risk. ${ }^{(23)}$ In the updated 2014 guidelines, the

\section{Table I. Statin-intensity approach to treatment. ${ }^{(22,23)}$}

\begin{tabular}{lccc}
\hline Parameter & \multicolumn{3}{c}{ Intensity level } \\
\cline { 2 - 4 } & High & Moderate & Low \\
\hline Statin (mg) & $20^{*}-80$ & $10-20^{*}$ & - \\
Atorvastatin & $20-40$ & $5-10$ & - \\
Rosuvastatin & - & $20-40$ & 10 \\
Simvastatin & - & $80(\times 2)$ & $20-40$ \\
Fluvastatin & - & 40 & 20 \\
Lovastatin & - & $40-80$ & $10-20$ \\
Pravastatin & - & $2-4$ & 1 \\
Pitavastatin & & & $<-<50$ \\
LDL reduction (\%) & $>40$ & $31-40$ & $20-30$ \\
ACC/AHA 2013(22) & 50 & 30 & \\
NICE 2014(23) & & & \\
\hline
\end{tabular}

*Atorvastatin $20 \mathrm{mg}$ is considered high-intensity according to the 2014 National Institute for Health and Care Excellence (NICE) guidelines, but is moderate-intensity according to the 2013 American College of Cardiology/ American Heart Association (ACC/AHA) guidelines. 
threshold for starting preventive treatment with statins has been reduced from a risk of $20 \%$ to $10 \%$ over ten years. ${ }^{(23)}$ The NICE guidelines also recommend that CVD risk should be assessed using the QRISK $2 ®$ assessment tool, which calculates the ten-year risks of $\mathrm{MI}$ and stroke based on 140,000 cardiovascular events in 2.3 million British patients (including different ethnic groups such as the Chinese and South Asians).

A recent review of cardiovascular assessment tools confirms that global risk assessment is essential for targeted, aggressive risk factor management. ${ }^{(24)}$ However, few CVD risk assessment tools have been developed in Asian countries for Asian populations, underscoring the need for an Asian-specific risk-assessment tool to accurately predict cardiovascular risk in these patients. ${ }^{(24)}$ Studies have found that the predictability of CVD risk scores, which were developed mainly for Caucasians, varies considerably, mainly due to overestimating the risks of $\mathrm{MI}$ in East Asians and underestimating lifetime risk among South Asians. ${ }^{(25)}$ It is probably safe to assume that the same is true for Singapore. A number of factors have been proposed to explain this. Firstly, data regarding incident CVD mortality, morbidity and risk factor prevalence in most East and South Asian populations is inadequate, thus preventing accurate calibration of a CVD risk score derived from another population. In addition, there is a lack of local longitudinal cohort data to supply robust information on the relationship between CVD risk factors and outcomes. ${ }^{(25)}$

\section{GUIDELINE RECOMMENDATIONS FOR ASCVD PREVENTION}

A number of local and international guidelines on CVD have been published. ${ }^{(22,23,26-29)}$ Each set of guidelines provides differing recommendations advocating a treat-to-target approach, a statin intensity-centric approach, or a combination of the LDL-C target and statin-centric approaches. In most of the current international guidelines, treatment targets have been eliminated; instead of a target-based approach, the new guidelines take a drug- and dose-based approach. The 2013 ACC/AHA cholesterol treatment guidelines emphasise that treatment decisions must be based on physician-patient discussion and that treatment may be indicated for some patients who are not in the four categories. ${ }^{(22)}$ The panel noted that with regard to key recommendations for statin therapy in the treatment and prevention of ASCVD, the role of statin therapy in the treatment paradigm for coronary artery disease differs among patient populations, and should therefore include both statin- and LDL-centric approaches, especially for patients in the high- and very-high-risk groups. ${ }^{(22)}$

\section{Statin- versus LDL-centric approach}

The 2013 ACC/AHA cholesterol guidelines adopt a statin-centric approach, disregarding LDL goals ${ }^{(22)}$ and discouraging the use of non-statin drugs that do not reduce the risk of events in statintreated patients. However, this approach has been challenged by the findings of the IMProved Reduction of Outcomes: Vytorin Efficacy International Trial (IMPROVE-IT), which investigated the simvastatin/ezetimibe combination in high-risk post-ACS patients and provided evidence that lower LDL-C levels were more beneficial (target LDL level $<55 \mathrm{mg} / \mathrm{dL}$ ). ${ }^{(30)}$ However, IMPROVE-IT showed that even with the addition of ezetimibe to simvastatin $40 \mathrm{mg}$, LDL-C levels decreased by only $40 \%$, whereas the guidelines recommend a reduction of at least $50 \%$ for this patient population. ${ }^{(22,23)}$

The revised 2015 American Diabetes Association (ADA) Standards of Medical Care in Diabetes guidelines have also adopted recommendations from the 2013 ACC/AHA guidelines for cholesterol management in diabetics, in which high-intensity statins were recommended for all diabetic patients with increased cardiovascular risk. ${ }^{(28)}$ In contrast, 2012 European guidelines for CVD prevention recommend treating to target cholesterol levels: $\mathrm{LDL}-\mathrm{C}<115 \mathrm{mg} / \mathrm{dL}(<3.0 \mathrm{mmol} / \mathrm{L})$ for subjects at low or moderate risk; LDL-C $<100 \mathrm{mg} / \mathrm{dL}(<2.5 \mathrm{mmol} / \mathrm{L})$ for high-risk patients; and LDL-C $<70 \mathrm{mg} / \mathrm{dL}(<1.8 \mathrm{mmol} / \mathrm{L})$ for very-highrisk patients. ${ }^{(26)}$ Furthermore, they recommend that patients with ACS should be started on high-intensity statin therapy in hospital.(26) The recently published 2015 European Society of Cardiology guidelines for ACS without persistent ST-segment elevation (NSTE-ACS), however, recommend the initiation of high-intensity statin therapy (i.e. statin regimens that reduce LDL-C by about $50 \%$ ) as early as possible after admission for all NSTE-ACS patients. ${ }^{(29)}$

The current Singapore clinical practice guidelines (CPG) on diabetes mellitus also advocate an LDL-centric approach for the prevention of ASCVD in diabetes mellitus. ${ }^{(27)}$ For the majority of patients with type 2 diabetes mellitus, where LDL-C is $>100 \mathrm{mg} / \mathrm{dL}$ ( $>2.6 \mathrm{mmol} / \mathrm{L}$ ), statin therapy should be started concurrently with therapeutic lifestyle modification. While the local guideline generally reflects the current practice, it also recognises that not all cases of diabetes mellitus should be considered a cardiovascular risk equivalent; it includes a category allowing for a period of lifestyle intervention without statin therapy in patients deemed to be at low risk. Although a number of recommendations are based on expert consensus, limited local objective evidence is available to support them. Based on the Singapore CPG on diabetes mellitus, in individuals without overt CVD, the goal is LDL-C $<100 \mathrm{mg} / \mathrm{dL}$, while in individuals with overt CVD or CKD, a lower LDL-C goal of $<82 \mathrm{mg} / \mathrm{dL}(2.1 \mathrm{mmol} / \mathrm{L})$ is advised. ${ }^{(27)}$ In a subgroup of patients with high LDL-C ( $>2.3 \mathrm{mmol} / \mathrm{L})$ and low HDL-C ( $<0.88 \mathrm{mmol} / \mathrm{L})$, statin-fenofibrate combination therapy may be beneficial. ${ }^{(27)}$

Despite the trend of population ageing, both within SEA and globally, there are no recommendations for the elderly aged $>75$ years or regarding the impact of the risk of CKD on prescribing decisions due to the lack of evidence in these patient populations. As guidelines are developed according to rigorous criteria such as requiring strong clinical trial evidence as a basis for recommendations, this lack of evidence prevented their development.

The Kidney Disease: Improving Global Outcomes (KDIGO) 2013 guidelines $^{(31)}$ highlight the limitations of LDL-C as a marker of cardiovascular risk in CKD and urge caution in using changes in LDL-C as a gauge for prescribing statins to patients with CKD. There is no on-treatment LDL-C target for CKD patients who are not on chronic dialysis or kidney transplantation; KDIGO 
recommends statin therapy as secondary ASCVD prevention for patients aged 18-49 years with one or more of the following: prior CHD; diabetes mellitus; or prior ischaemic stroke. A statin with or without ezetimibe, according to age, diabetes mellitus and estimated glomerular filtration rate (eGFR), is recommended for the primary prevention of ASCVD. ${ }^{(31)}$

\section{Current treatment practice in Singapore: are clinicians underdosing high-risk patients?}

In clinical trials, such as the Incremental Decrease in End Points Through Aggressive Lipid Lowering and Treating to New Targets studies, ${ }^{(32,33)}$ different doses of statins were studied and LDL-C levels were evaluated to ensure that the correct dose was used to avoid undertreatment. Most of the studies selected individuals with an existing clinical condition and treated them with fixeddose statins (e.g. high- vs. low-intensity or statin vs. placebo). None of the trials targeted a specific LDL-C level and there was no provision for dose up-titration. However, in clinical practice, there is a strong reluctance toward treatment based on statin intensity (e.g. high-intensity statins are better than low-intensity statins [Table I]), as opposed to the LDL-centric approach.

In Asia, there are many challenges in adopting the ACC/AHA 2013 guidelines. Evidence indicates that Chinese patients have a higher incidence of myopathy at much lower statin doses. ${ }^{(34)}$ Consequently, there is reluctance to use high-intensity statins at the maximum dose. The ACC/AHA Pooled Cohort Equations risk assessment is unsuitable for Asian populations, as patients from this region were not included during the development of the tool. ${ }^{(22)}$ Recent guidelines also recommend treating risk rather than LDL-C levels. ${ }^{(22,23)}$ In Singapore, there are many high-risk patients who are not on high-intensity statins, as there is a perception that Asians are intolerant to statins. ${ }^{(35)}$ Anecdotal evidence suggests that in Singapore, there is concern among both physicians and patients regarding intensifying statins; both groups are averse to using highintensity statins and prefer dose reduction once LDL-C levels fall below $70 \mathrm{mg} / \mathrm{dL}$. Increased understanding of the pathogenesis of ASCVD has underscored the importance of treating both risk factors and LDL-C levels. Clinicians should consider the evidence in favour of reducing LDL-C levels in young, high-risk individuals with heterozygous familial hypercholesterolaemia in order to achieve maximal long-term cardiovascular benefits. However, it is a challenge to convince Asian doctors and patients to adopt this approach.

\section{Asians are more susceptible to adverse effects of high-intensity statins: evidence or opinion?}

Available data indicates that the effect of statins in Asian patients is not a class effect. For atorvastatin, similar pharmacokinetics in Asians and Caucasians predict a correlation between efficacy and safety in Asian and global studies, supporting similar dosing regimens in these populations. ${ }^{(36)}$ In contrast, systemic exposure to rosuvastatin is higher in Asians compared with Caucasians; ${ }^{(37)}$ significant differences in pharmacokinetics between these patient populations predict a lack of correlation between efficacy and safety. Consequently, prescribing information for rosuvastatin recommends the same low starting dose (5 mg) in Asians as in patients with renal impairment. ${ }^{(38)}$ Emerging data also suggests that rosuvastatin may have an adverse effect on renal function, with significant reductions in eGFR observed in patients with type 1 or 2 diabetes mellitus. ${ }^{(39)}$

Evidence confirms that the adverse effects of atorvastatin are similar across the dose range. ${ }^{(36,37)}$ The Insulin Resistance Intervention after Stroke trial involving patients of South Asian origin with $\mathrm{CHD}$ also showed a dose-dependent reduction in LDL-C with atorvastatin and a comparable safety profile in both arms of the study (i.e. atorvastatin $10 \mathrm{mg}$ and $20 \mathrm{mg}$ ). ${ }^{(36)}$ Unlike atorvastatin, simvastatin was associated with a higher risk of myopathy in a large cohort of Chinese patients, as compared with Caucasian patients $(n=25,673) .{ }^{(34)}$ The absolute risk of any myopathy (definite or incipient) was much higher in China than in Europe (0.13\%/year vs. 0.04\%/year; $p=0.001)$. Recently reported findings from the Heart Protection Study 2 Treatment of HDL to Reduce the Incidence of Vascular Events (HPS2-THRIVE), which showed a higher rate of myopathy in the Chinese population, ${ }^{(34)}$ have led to new precautions, including advising caution when prescribing simvastatin to Asian patients and that the lowest dose necessary should be employed. Although Chinese patients were the only Asians assessed in HPS2-THRIVE and data on other Asian populations is limited, the manufacturer has proposed that these new precautions for simvastatin use be extended to all Asians, including Japanese, Malays and Asian-Indians, due to the potential seriousness of myopathy. Therefore, the Health Sciences Authority, Singapore, has recommended updating local package inserts of products containing simvastatin to provide new recommendations for the use of simvastatin in Asians. ${ }^{(40)}$

\section{Expert opinions and recommendations for ideal clinical practice in Singapore}

There are currently a number of international and local guidelines that advocate different approaches to ASCVD prevention and management. ${ }^{(22,23,26-29)}$ The local CPG on diabetes mellitus remains LDL-centric, ${ }^{(27)}$ in contrast to recent guidelines, such as ACC/AHA 2013 and ADA 2015, that recommend high-intensity statins for primary prevention in high-risk diabetic patients. ${ }^{(22,28)}$ NICE 2014 recommends atorvastatin $20 \mathrm{mg}$ (i.e. high-intensity according to NICE) for the primary prevention of CVD and atorvastatin $80 \mathrm{mg}$ for secondary prevention in patients with CVD. ${ }^{(23)}$ In Singapore, there is a clear need for a standard multidisciplinary consensus on lipid-lowering treatment that is specific to the local population and includes the modified Singapore Framingham risk score. Meanwhile, this expert panel identified some common principles to consider when treating patients at risk of ASCVD.

\section{Risk prediction scoring tool}

Assessment of ASCVD risk is important and Asian-specific risk assessment tools (risk calculators) should be used when available. Anecdotal evidence suggests that the ASCVD risk calculator is underutilised among Asians. Ideally, each country should develop a risk calculator that is applicable to its 
population or recalibrate existing international risk calculators. If one is not available, the QRISK2 calculator may be a better option for Asians than the ACC/AHA Pooled Cohort Equations, as it incorporates Asian ethnic groups (e.g. Chinese, Indian, Pakistani, Bangladeshi and other Asians), CKD, atrial fibrillation, rheumatoid arthritis and body mass index. ${ }^{(23)}$ The JBS3 calculator, produced by the Joint British Societies for the prevention of CVD, included estimations of lifetime risk, which may enable a more accurate ASCVD risk assessment. ${ }^{(41)}$ However, the effectiveness of the JBS3 calculator to assess risk in the Asian population remains to be determined.

\section{Lifestyle modification}

The panel concurred that lifestyle modifications may be employed in patients at low risk of CVD. This recommendation is in agreement with the local guidelines, which allow for a period of lifestyle intervention without statin therapy in patients who are considered low-risk. ${ }^{(28)}$

\section{Low- versus high-intensity statin}

Ideally, clinicians should aim for LDL-C targets based on the National Cholesterol Education Program's Adult Treatment Panel III guidelines. ${ }^{(42)}$ However, as this approach may result in undertreatment of many patients, a risk-based approach may be more appropriate. Furthermore, it is important to start statin therapy at the intended dose. In the Asian population, atorvastatin 10-20 mg is suitable for primary prevention, while 40-80 mg should be considered for secondary prevention (achieving $>50 \%$ LDL-C reduction). Rosuvastatin $10 \mathrm{mg}$ and $20 \mathrm{mg}$ (up to $40 \mathrm{mg}$ ) can also be considered for primary and secondary prevention, respectively. Higher statin doses that will produce lower LDL-C levels should be adhered to in patients with overt CVD. Commencing statins and continuing on longterm statin therapy in younger high-risk individuals (e.g. those with heterozygous familial hypercholesterolaemia) may confer greater cardiovascular benefits than initiating treatment at an older age. Evidence shows that atorvastatin has a similar tolerability profile in Asian and Caucasian patients. However, consideration should be given to the recent findings of the HPS2-THRIVE study when prescribing high-intensity simvastatin to Asian patients.

Based on the above, it is evident that the Singapore ASCVD expert group has assimilated the most appropriate recommendations from the various current guidelines for the following population subgroups (Table II):

1. Patients with established ASCVD, e.g. CHD, history of $\mathrm{MI}$, stable angina, ACS (i.e. unstable angina, non-ST-segment elevation MI and ST-segment elevation MI), stroke, transient ischaemic attack and peripheral arterial disease, as secondary prevention.

(a) High-intensity statins are usually required to achieve maximal cholesterol lowering of $\geq 50 \%$ LDL-C reduction, although it is recognised that there may be inter-individual variations in the response to LDL-C reduction with a fixed dose of statin. (b) Atorvastatin 40-80 mg or rosuvastatin 20-40 mg reduces LDL-C by an average of $\geq 50 \%$.

(c) There is still a role for an LDL-centric approach; LDL-C should be lowered to very low levels (preferably $\leq 70 \mathrm{mg} / \mathrm{dL}$ ).

(d) Based on current evidence, ${ }^{(30,43)}$ in very-high-risk patients who are unable to achieve $\geq 50 \%$ LDL-C reduction or are not on LDL target $\leq 70 \mathrm{mg} / \mathrm{dL}$, the addition of ezetimibe can be considered; recently, proprotein convertase subtilisin/kexin type 9 (PCSK9) monoclonal antibodies have also been recommended. ${ }^{(43,44)}$

2. Patients with familial hypercholesterolaemia, with LDL-C $\geq 190 \mathrm{mg} / \mathrm{dL}$.

(a) High-intensity statins are required to lower LDL-C as much as possible.

(b) The addition of a non-statin drug (e.g. ezetimibe) may be required to further lower LDL-C levels. These recommendations are based on the fact that individuals with LDL-C $\geq 190 \mathrm{mg} / \mathrm{dL}$, even without risk factors, have a high lifetime risk of ASCVD.

(c) PCSK9 inhibitors have recently received the approval of the United States Food and Drug Administration for the treatment of familial hypercholesterolaemia. ${ }^{(44)}$

3. Diabetes mellitus (type 1 or 2 ) patients aged $40-75$ years, with LDL-C of 70-189 mg/dL.

(a) There is almost universal agreement that diabetic patients with established ASCVD should receive high-intensity statin therapy to achieve maximal LDL-C lowering of $\geq 50 \%$ in order to attain very low levels of LDL-C ( $\leq 70 \mathrm{mg} / \mathrm{dL}) .{ }^{(23)}$ Based on the current consensus, if patients cannot achieve $\geq 50 \%$ LDL-C reduction or are not on an LDL-C target $\leq 70$ $\mathrm{mg} / \mathrm{dL}$, the addition of ezetimibe or PCSK9 inhibitors can be considered.

(b) Primary prevention

i. In patients who are categorised as high-risk (ASCVD pool cohort $\geq 7.5 \%$ or QRISK2/JBS3 $\geq 10 \%$ ), highintensity statin therapy can be considered. For those in the moderate-risk category (ASCVD $<7.5 \%$ or QRISK2/ JBS3 $<10 \%$ ), moderate-intensity statin therapy can be considered.

ii. For patients with a residual risk burden (especially high triglycerides $\geq 200 \mathrm{mg} / \mathrm{dL}, \mathrm{HDL}-\mathrm{C}<40 \mathrm{mg} / \mathrm{dL}$ ), the addition of a fibrate after the commencement of statin therapy can be considered.

4. Patients aged $40-75$ years, with LDL-C of $70-189 \mathrm{mg} / \mathrm{dL}$, as primary prevention.

(a) Individuals in this category have numerous complex issues depending on their risk profile.

(b) Global risk assessment using the risk calculator is required, with categorisation into high- or moderate-risk for initiation of high- or moderate-intensity statin therapy (refer to 3bi \& ii).

5. CKD patients

(a) Assessment and classification of CKD stage is required, using the KDIGO eGFR nomenclature. ${ }^{(28)}$ 
Table II. Key recommendations for treatment of blood cholesterol to reduce and prevent atherosclerotic cardiovascular disease (ASCVD) risk in adults.

\begin{tabular}{|c|c|c|c|c|c|c|c|c|c|}
\hline Category & \multicolumn{2}{|c|}{$\begin{array}{c}\text { 1) Clinical ASCVD } \\
\text { (CHD, history of MI, stable angina, UA, } \\
\text { ACS, NSTEMI, STEMI, stroke, TIA, PAD) }\end{array}$} & $\begin{array}{l}\text { 2) Familial } \\
\text { hypercholesterolaemia } \\
\text { LDL-C } \geq 190 \mathrm{mg} / \mathrm{dL}\end{array}$ & \multicolumn{3}{|c|}{$\begin{array}{l}\text { 3) DM type } 1 \text { or } 2 \\
\text { LDL-C } 70-189 \mathrm{mg} / \mathrm{dL}\end{array}$} & \multicolumn{2}{|c|}{$\begin{array}{l}\text { 4) Primary prevention } \\
\text { No DM/clinical ASCVD } \\
\text { LDL-C } 70-189 \mathrm{mg} / \mathrm{dL}\end{array}$} & $\begin{array}{l}\text { 5) CKD (G3-5)* } \\
\text { Patients on } \\
\text { dialysis or with } \\
\text { kidney transplant }\end{array}$ \\
\hline Age (yr) & $\leq 75$ & $>75$ & $\geq 21$ & \multicolumn{3}{|c|}{$40-75$} & \multirow{2}{*}{\multicolumn{2}{|c|}{$\begin{array}{c}40-75 \\
\text { Primary } \\
\text { (without clinical ASCVD) }\end{array}$}} & $>50$ \\
\hline Prevention & $\begin{array}{c}\text { Secondary } \\
\text { (clinical ASCVD) }\end{array}$ & $\begin{array}{c}\text { Secondary } \\
\text { (clinical ASCVD) }\end{array}$ & $\begin{array}{l}\text { Primary (without } \\
\text { clinical ASCVD) }\end{array}$ & $\begin{array}{c}\text { Secondary } \\
\text { (clinical ASCVD) }\end{array}$ & $\begin{array}{r}\text { Pri } \\
\text { (without cli }\end{array}$ & $\begin{array}{l}\text { hary } \\
\text { ical ASCVD) }\end{array}$ & & & Primary/secondary \\
\hline \multirow[t]{2}{*}{ Global risk assessment } & \multirow[t]{2}{*}{ Not required } & \multirow[t]{2}{*}{ Not required } & \multirow[t]{2}{*}{ Not required } & \multirow[t]{2}{*}{ Not required } & \multicolumn{2}{|c|}{ Risk assessment required $^{+}$} & \multicolumn{2}{|c|}{ Risk assessment required ${ }^{+}$} & Not required \\
\hline & & & & & High risk & Moderate risk & High risk & Moderate risk & \\
\hline \multicolumn{10}{|l|}{ LDL-centric approach } \\
\hline LDL-goal target (mg/dL) & $<60$ to 70 & 70 to $<100$ & $\begin{array}{l}\text { As low as possible } \\
\qquad(<100)\end{array}$ & $\begin{array}{l}<60 \text { to } 70 \\
<80^{(27)}\end{array}$ & $\begin{array}{l}<70 \\
<80^{(27)}\end{array}$ & $<100$ & $<70$ & $<100$ & No LDL target \\
\hline LDL reduction (\%) & $\geq 50$ & 30 to $<50$ & $\geq 50$ & $\geq 50$ & $\geq 50$ & 30 to $<50$ & $\geq 50$ & 30 to $<50$ & 30 to $<50$ \\
\hline \multicolumn{10}{|l|}{ Statin-centric approach } \\
\hline Intensity & High & Moderate & High & High & High & Moderate & Moderate to high & Moderate & Moderate \\
\hline \multicolumn{10}{|l|}{ Type of statin dose* $(\mathrm{mg})$} \\
\hline Atorvastatin & $40-80(\mathrm{H})$ & $10-20(\mathrm{M})$ & $40-80(\mathrm{H})$ & $40-80(\mathrm{H})$ & $40-80(\mathrm{H})$ & $10-20(M)$ & $\begin{array}{l}10-20(\mathrm{M}) \\
40-80(\mathrm{H})\end{array}$ & $10-20(M)$ & $10-20(M)$ \\
\hline Rosuvastatin & $20-40(\mathrm{H})$ & $5-10(\mathrm{M})$ & $20-40(\mathrm{H})$ & $20-40(\mathrm{H})$ & $20-40(\mathrm{H})$ & $5-10(\mathrm{M})$ & $\begin{array}{l}5-10(\mathrm{M}) \\
20-40(\mathrm{H})\end{array}$ & 5-10 (M) & $5-10(\mathrm{M})$ \\
\hline Simvastatin & - & $20-40(\mathrm{M})$ & - & - & - & $20-40(\mathrm{M})$ & $20-40(\mathrm{M})$ & $20-40(\mathrm{M})$ & $20-40(\mathrm{M})$ \\
\hline Pravastatin & - & $40-80(\mathrm{M})$ & - & - & - & $40-80(\mathrm{M})$ & 40-80 (M) & $40-80(\mathrm{M})$ & $40(\mathrm{M})$ \\
\hline Fluvastatin XL & - & $80(\mathrm{M})$ & - & - & - & $80(\mathrm{M})$ & $80(\mathrm{M})$ & $80(\mathrm{M})$ & $80(\mathrm{M})$ \\
\hline Lovastatin & - & $40(\mathrm{M})$ & - & - & - & $40(\mathrm{M})$ & $40(\mathrm{M})$ & $40(\mathrm{M})$ & No data \\
\hline Pitavastatin & - & $2-4(M)$ & - & - & - & $2-4(\mathrm{M})$ & $2-4(M)$ & $2-4(M)$ & $2(\mathrm{M})$ \\
\hline \multicolumn{10}{|l|}{ Add non-statin } \\
\hline Ezetimibe & Yes & Yes & Yes & - & - & - & - & - & Yes \\
\hline PCSK9 inhibitor & Can consider & - & Yes & Can consider & - & - & - & - & - \\
\hline
\end{tabular}

*M: moderate-intensity statin therapy, H: high-intensity statin therapy. tRisk assessment with ASCVD Pooled Cohort Equation - high risk: $\geq 7.5 \%$, moderate risk: 5 to $<7.5 \% ;$ QRISK2/JBS3 - high risk: $\geq 10 \%$, moderate risk: $<10 \%$. ₹KDIGO chronic kidney disease (CKD) nomenclature (eGFR mL/min/1.73 $\mathrm{m}^{2}$ ) - G1: normal, G2: mildly decreased 60-89, G3: 30-59, G4: 15-29, G5 < 15. ACS: acute coronary syndrome; CHD: coronary heart disease; DM: diabetes mellitus; LDL-C: low-density lipoprotein cholesterol; MI: myocardial infarction; NSTEMI: non-ST-segment elevation myocardial infarction; PAD: peripheral arterial disease; PCSK9: proprotein convertase subtilisin/kexin type 9; STEMI: ST-segment elevation myocardial infarction; TIA: transient ischaemic attack; UA: unstable angin 
(b) LDL-C targets should be avoided due to the poor correlation of LDL-C levels with CVD risk. There is a paradoxical association between cholesterol and cardiovascular outcomes, especially in patients with end-stage renal disease who require dialysis, due to confounding factors such as malnutrition, inflammation and the effect of modification by protein energy wasting, which lower the LDL-C. Additionally, advanced kidney failure patients with low cholesterol, who are at high risk, are often not identified.

(c) Statin-naïve patients on dialysis do not appear to benefit from the initiation of statin therapy (similar to heart failure Class II-IV).

(d) All CKD patients who are not on dialysis are at high risk for ASCVD and should be given moderate-intensity statin therapy (refer to $3 \mathrm{bi} \& \mathrm{ii}$ ). Dialysis and renal transplant patients who are already on statin therapy should remain on it.

(e) Moderate-intensity statins are recommended for advanced CKD patients to avoid the high risk of medication-related adverse events because of reduced renal excretion, frequent polypharmacy and high prevalence of comorbidities.

(f) Combination therapy with a non-statin drug (e.g. ezetimibe) is useful for avoiding high-intensity statin therapy to attain the desired LDL-C reduction.

(g) Statin therapy is appropriate in adult kidney transplant recipients. However, toxicity due to drug interactions, especially with calcineurin inhibitors, needs to be taken into account. ${ }^{(45)}$ A low starting dose with cautious increments, according to patient tolerability and indication, is recommended.

\section{CONCLUSION}

Increased understanding of the pathogenesis of ASCVD has underscored the importance of treating both risk factors and LDL-C levels. Recent guidelines are in favour of treating CVD risks rather than targeting specific LDL-C levels. At present, there are no consolidated guidelines and risk assessment tools for ASCVD management in Singapore. Hence, physicians must rely on clinical judgement to make best practice decisions for their patients. Additionally, there are concerns among both physicians and patients in Singapore regarding intensifying statins, as there is a perception that Asians are less tolerant to high-intensity statins. Consequently, many high-risk patients are not on high-intensity statins, despite evidence confirming that the efficacy and safety of certain statins (e.g. atorvastatin) are similar in Asian and Caucasian patients across the dose range. Therefore, a locally developed and validated risk assessment tool is needed to guide treatment decisions for Singapore physicians.

\section{ACKNOWLEDGEMENTS}

The authors acknowledge Anna Battershill and See Mee Yen from Medica Comms Pte Ltd for providing editorial support, which was funded by Pfizer Pte Ltd, Singapore.

\section{CONFLICT OF INTEREST}

All authors received honoraria for their participation in the expert panel meeting on Primary and Secondary Prevention of Atherosclerotic Cardiovascular Disease (ASCVD) in Adults Current Clinical Evidence and Practice on 16 August 2014, funded by Pfizer Pte Ltd. The authors did not receive compensation for their authorship of this article.

\section{MEMBERS OF THE SINGAPORE ASCVD GROUP}

Peter Yan (Singapore), Kevin Tan Eng Kiat (Singapore), Jason Choo Chon Jun (Singapore), Stanley Liew Choon Fong (Singapore), Titus Lau Wai Leong (Singapore), David D Waters (USA), Tan Swee Yaw* (Singapore), Ho Chee Khun* (Singapore).

*not involved as authors of this manuscript

\section{REFERENCES}

1. Yusuf S, Reddy S, Ounpuu S, Anand S. Global burden of cardiovascular diseases: Part II: variations in cardiovascular disease by specific ethnic groups and geographic regions and prevention strategies. Circulation 2001; 104:2855-64.

2. Yusuf S, Reddy S, Ounpuu S, Anand S. Global burden of cardiovascular diseases: part I: general considerations, the epidemiologic transition, risk factors, and impact of urbanization. Circulation 2001; 104:2746-53.

3. Tai ES, Poulton R, Thumboo J, et al. An update on cardiovascular disease epidemiology in South East Asia. Rationale and design of the LIFE course study in CARdiovascular disease Epidemiology (LIFECARE). CVD Prevention Control 2009; 4:93-102.

4. World Health Organization. Noncommunicable Diseases in the South-East Asia Region 2011: Situation and Response. Available at: http://www.searo. who.int/nepal/mediacentre/2011_non_communicable_diseases_in_the_ south_east_asia_region.pdf. Accessed August 28, 2015.

5. Ueshima H, Sekikawa A, Miura K, et al. Cardiovascular disease and risk factors in Asia: a selected review. Circulation 2008; 118:2702-9.

6. World Health Organization. CVD Atlas 2002: Deaths from stroke. Available at: http://www.who.int/cardiovascular_diseases/en/cvd_atlas_16_death_ from_stroke.pdf. Accessed August 28, 2015.

7. World Health Organization. CVD Atlas 2002: Deaths from coronary heart disease. Available at: www.who.int/cardiovascular_diseases/en/ cvd_atlas_14_deathHD.pdf. Accessed August 28, 2015.

8. Phan TP, Alkema L, Tai ES, et al. Forecasting the burden of type 2 diabetes in Singapore using a demographic epidemiological model of Singapore. BMJ Open Diabetes Res Care 2014; 2:e00012.

9. Moran A, Gu D, Zhao D, et al. Future cardiovascular disease in China: Markov model and risk factor scenario projections from the coronary heart disease policy model-China. Circ Cardiovasc Qual Outcomes 2010; 3:243-52.

10. Zhang X, Patel A, Horibe H, et al; Asia Pacific Cohort Studies Collaboration. Cholesterol, coronary heart disease, and stroke in the Asia Pacific region. Int J Epidemiol 2003; 32:563-72.

11. Wu CC, Sy R, Tanphaichitr V, et al. Comparing the efficacy and safety of atorvastatin and simvastatin in Asians with elevated low-density lipoprotein-cholesterol--a multinational, multicenter, double-blind study. J Formos Med Assoc 2002; 101:478-87.

12. Chan $P$, Huang TY, Tomlinson B, Lee C, Lee YS. Short-term safety and efficacy of low-dose simvastatin in elderly patients with hypertensive hypercholesterolemia and fasting hyperinsulinemia. J Clin Pharmacol 1997; 37:496-501.

13. Efficacy of atorvastatin in primary hypercholesterolemia. Japan Cholesterol Lowering Atorvastatin Study (J-CLAS) Group. Am J Cardiol 1997; 79:1248-52.

14. Saito Y, Goto Y, Dane A, Strutt K, Raza A. Randomized dose-response study of rosuvastatin in Japanese patients with hypercholesterolemia. Atheroscler Thromb 2003; 10:329-36.

15. Wang KY, Ting CT. A randomized, double-blind, placebo-controlled, 8-week study to evaluate the efficacy and safety of once daily atorvastatin (10 mg) in patients with elevated LDL-cholesterol. Jpn Heart J 2001; 42:725-38.

16. Yamamoto A, Arakawa K, Sasaki J, et al; Rosuvastatin Dose-Ranging Trialist Group. Clinical effects of rosuvastatin, a new HMG-CoA reductase 
inhibitor, in Japanese patients with primary hypercholesterolemia: an early phase II study. J Atheroscler Thromb 2002; 9:48-56.

17. Yan P. Cardiovascular Disease Risk Management in Diabetes - The Implications of the REALITY Asia Study. Available at: http://www. touchophthalmology.com/sites/www.touchoncology.com/files/migrated/ articles_pdfs/yan.pdf. Accessed September 25, 2015.

18. Kim HS, Wu Y, Lin SJ, et al. Current status of cholesterol goal attainment after statin therapy among patients with hypercholesterolemia in Asian countries and region: the Return on Expenditure Achieved for Lipid Therapy in Asia (REALITY-Asia) study. Curr Med Res Opin 2008; 24:1951-63.

19. Ho KT, Chin KW, Ng KS, et al. The A-SACT (Achievement in Singapore of Cholesterol Targets) study in patients with coronary heart disease. Am J Cardiovasc Drugs 2006; 6:383-91.

20. Chin CW, Gao F, Le T, Tan R. Lipid goal attainment and prescription behavior in Asian patients with acute coronary syndromes: experience from a tertiary hospital. Clin Med Insights Cardiol 2013; 7:51-7.

21. Waters DD, Brotons C, Chiang CW, et al; Lipid Treatment Assessmen Project 2 Investigators. Lipid treatment assessment project 2: a multinational survey to evaluate the proportion of patients achieving low-density lipoprotein cholesterol goals. Circulation 2009; 120:28-34.

22. Stone NJ, Robinson JG, Lichtenstein $\mathrm{AH}$, et al; American College of Cardiology/American Heart Association Task Force on Practice Guidelines. 2013 ACC/AHA Guideline on the Treatment of Blood Cholesterol to Reduce Atherosclerotic Cardiovascular Risk in Adults: a report of the American College of Cardiology/American Heart Association Task Force on Practice Guidelines. Circulation 2014; 129 (25 Suppl 2):S1-45.

23. Rabar S, Harker M, O'Flynn N, Wierzbicki AS; Guideline Development Group. Lipid modification and cardiovascular risk assessment for the primary and secondary prevention of cardiovascular disease: summary of updated NICE guidance. BMJ 2014; 349:g4356.

24. Liau SY, Izham MI, Hassali MA, Shafie AA. A literature review of the cardiovascular risk-assessment tools: applicability among Asian population. Heart Asia 2010; 2:15-8.

25. Hussain SM, Oldenburg B, Wang Y, Zoungas S, Tonkin AM. Assessmen of cardiovascular disease risk in South Asian populations. Int J Vasc Med 2013; 2013:786801.

26. Perk J, De Backer G, Gohlke H, et al; European Association for Cardiovascular Prevention \& Rehabilitation (EACPR); ESC Committee for Practice Guidelines (CPG). European Guidelines on cardiovascular disease prevention in clinical practice (version 2012). The Fifth Joint Task Force of the European Society of Cardiology and Other Societies on Cardiovascular Disease Prevention in Clinical Practice (constituted by representatives of nine societies and by invited experts). Eur Heart J 2012; 33:1635-701.

27. Ministry of Health, Singapore. Diabetes Mellitus Clinical Practice Guidelines. Available at: http://www.moh.gov.sg/content/moh_web/ healthprofessionalsportal/doctors/guidelines/cpg_medical/2014/cpgmed_ diabetes_mellitus.html. Accessed August 29, 2015.

28. America Diabetes Association. Standards of Medical Care in Diabetes 2015. Diabetes Care 2015; 38(Suppl 1):S41-S48.

29. Roffi M, Patrono C, Collet JP, et al. 2015 ESC Guidelines for the management of acute coronary syndromes in patients presenting without persistent ST-segment elevation: Task Force for the Management of Acute Coronary Syndromes in Patients Presenting without Persistent ST-Segment Elevation of the European Society of Cardiology (ESC). Eur Heart J 2016; 37:267-315.

30. Cannon CP, Blazing MA, Giugliano RP, et al; IMPROVE-IT Investigators. Ezetimibe Added to Statin Therapy after Acute Coronary Syndromes. N Engl J Med 2015; 372:2387-97.
31. Pharmacological cholesterol-lowering treatment in adults. In: KDIGO Clinical Practice Guideline for Lipid Management in Chronic Kidney Disease. Kidney Int Supplements 2013; 3:271-9.

32. Pedersen TR, Faergeman O, Kastelein JJ, et al; Incremental Decrease in End Points Through Aggressive Lipid Lowering (IDEAL) Study Group. High-dose atorvastatin vs usual-dose simvastatin for secondary prevention after myocardial infarction: the IDEAL study: a randomized controlled trial. JAMA 2005; 294:2437-45.

33. LaRosa JC, Grundy SM, Kastelein JJ, Kostis JB, Greten H; Treating to New Targets (TNT) Steering Committee and Investigators. Safety and efficacy of Atorvastatin-induced very low-density lipoprotein cholesterol levels in Patients with coronary heart disease (a post hoc analysis of the treating to new targets [TNT] study). Am J Cardiol 2007; 100:747-52.

34. HPS2-THRIVE Collaborative Group. HPS2-THRIVE randomized placebocontrolled trial in 25673 high-risk patients of ER niacin/laropiprant: trial design, pre-specified muscle and liver outcomes, and reasons for stopping study treatment. Eur Heart J 2013; 34:1279-91.

35. Setia S, Fung SS, Waters DD. Doctors' knowledge, attitudes, and compliance with 2013 ACC/AHA guidelines for prevention of atherosclerotic cardiovascular disease in Singapore. Vasc Health Risk Manag 2015; 11:303-10.

36. Gandelman K, Fung GL, Messig M, Laskey R. Systemic exposure to atorvastatin between Asian and Caucasian subjects: a combined analysis of 22 studies. Am J Ther 2012; 19:164-73.

37. Lee E, Ryan S, Birmingham B, et al. Rosuvastatin pharmacokinetics and pharmacogenetics in white and Asian subjects residing in the same environment. Clin Pharmacol Ther 2005; 78:330-41.

38. Crestor (Rosuvastatin) Singapore Prescribing Information. November 2012. Available at: http://eservice.hsa.gov.sg/prism/common/enquirepublic/ SearchDRBProduct.do?action=getProductDetails. Accessed August 29, 2015.

39. de Zeeuw D, Anzalone DA, Cain VA, et al. Renal effects of atorvastatin and rosuvastatin in patients with diabetes who have progressive renal disease (PLANET I): a randomised clinical trial. Lancet Diabetes Endocrinol 2015; 3:181-90.

40. Health Sciences Authority, Singapore. 29th August 2014: New recommendations for simvastatin use in Asian based on findings from the HPS2-THRIVE study. Available at: www.hsa.gov.sg/content/hsa/en/ Health_Products_Regulation/Safety_Information_and_Product_Recalls/ Product_Safety_Alerts/2014/new_recommendations_for_simvastatin_use. html. Accessed August 29, 2015.

41. Joint British Societies for the Prevention of Cardiovascular Disease. Risk Calculator. Available at: http://www.jbs3risk.com/pages/risk_calculator. htm. Accessed October 4, 2015.

42. National Cholesterol Education Program. ATP III Guidelines At-a-Glance Quick Desk Reference. Available at: http://www.nhlbi.nih.gov/files/docs/ guidelines/atglance.pdf. Accessed August 29, 2015.

43. Lloyd-Jones DM, Morris PB, Ballantyne CM, et al. 2016 ACC Expert Consensus Decision Pathway on the Role of Non-Statin Therapies for LDLCholesterol Lowering in the Management of Atherosclerotic Cardiovascular Disease Risk: A Report of the American College of Cardiology Task Force on Clinical Expert Consensus Documents. J Am Coll Cardiol 2016; pii: S0735-1097(16)32398-1.

44. Everett BM, Smith RJ, Hiatt WR. Reducing LDL with PCSK9 Inhibitors--The Clinical Benefit of Lipid Drugs. N Engl J Med 2015; 373:1588-91.

45. Magee CC, Pascual M. Update in renal transplantation. Arch Intern Med 2004; $164: 1373-88$ 